Review Article

\title{
HUMAN CANCER IS A PARASITE SPREAD VIA INTRUSION IN GENOME
}

\author{
Sergey N. Rumyantsev \\ Department of Evolutionary Immunology \\ Andent, Inc., New Jersey, 07302 USA \\ rumyan1@yahoo.com
}

\begin{abstract}
The present article is devoted to further development of new paradigm about the biology of human cancer: the hypothesis of parasitic nature, origin and evolution of the phenomenon. The study included integrative reconsidering, and reinterpretation of the make-ups, traits and processes existing both in human and animal cancers. It was demonstrated that human cancer possesses nearly analogous set of traits characteristic of transmissible animal cancer. Undoubted analogies are seen in the prevalence, clinical exposure, progression of disease, origin of causative agents, immune response against invasion and especially in the intrinsic deviations of the leading traits of cancerous cells. Both human and animal cancers are highly exceptional pathogens. But in contrast to contagious animal cancers the cells of of human cancer can not pass between individuals as usual infectious agents. Exhaustive evidence of the parasitic nature and evolutionary origin of human cancer was revealed and interpreted. In contrast to animal cancer formed of solitary cell lineage, human cancer consists of a couple of lineages constructed under different genetic regulations and performed different structural and physiological functions. The complex make-up of cancer composition remains stable over sequential propagation. The subsistence of human cancer regularly includes obligatory interchange of its successive forms. Human cancer possesses its own biological watch and the ability to gobble its victim, transmit via the intrusion of the genome, perform intercommunications within the tumor components and between the dispersed subunits of cancer. Such intrinsic traits characterize human cancer as a primitively structured parasite that can be classified in Class Mammalians, Species Genomeintruder malevolent (G.malevolent).
\end{abstract}

Keywords: Cancer ecology; Cancer immunology; Cancerous genome; Cancerous parasitism; Genomic intrusion;

\section{Introduction}

For many centuries cancer killed only some people. But at 1940 the spread of cancer overtook many infectious diseases as an important human killer. Today it is one of the biggest threats to global human health. Nevertheless, human cancer, unlike kinds of animal cancer, was unconditionally considered as non-transmissible. This hypothetical statement was supported in a plethora of medical articles, monographs, dictionaries, encyclopedias and websites. Over 80 years any case cancer disease has been considered as a result of individually happened somatic mutation of an alone cell and subsequent dispersion of its cancerous offspring around afflicted human body. Recently, the unanimity of judgment about the non-transmissibility of human cancer has been disturbed by publications [1, 2] that proposed principally a new paradigm of invasive parasitic nature, transmission, origin, evolution, and pathogenesis of human cancer. At least four different kinds of transmissible cancerous malignancies have now been identified in humans and animals. Firstly, some forms of malignancies can arise in some humans from infection from specific contagious viruses or bacteria [3]. Secondly, there exist various forms of transmissible malignant tumor among animals [4]. Thirdly, there are tumors that are hypothetically identified as transferrable from mother to fetus [5] as well as cancer initiated in one twin fetus and spread to the co-twin via vascular anastomoses within the placenta [6]. Finally, predominant human cancers should now be considered as transmissible too [7-10]. In the present article, the new paradigm [1,2] is developed mainly by supplementing and supporting its core principles with some very important data which could not be considered before. The search includes mainly 
the results of integrative reconsidering, systematization and reinterpretation of both the known and recent data concerning the ways of transmission, immunology, genetics, pathogenesis and clinical exposures of natural cancer in not only humans but also in animals.

\section{Materials and methods}

The present article is devoted to the development of the new paradigm by supplementing its core principles with the set of data which has not been considered before. The search was performed by multidisciplinary reconsidering, systematization, integration and reinterpretation of both known and recent data concerning the ways of transmission, immunology, genetics, pathogenesis and clinical exposures of both human and animal cancer.

\section{Results and discussion}

\subsection{Origin of animal cancer}

The existence of contagious cancer is well known among dogs and other canines, [4] Tasmanian devils, [11] Syrian hamster, [12, 13] sea lions (genital carcinoma), sea turtles (fibropapillomatosis), beluga whales, bottom-dwelling fish [3, 14] and so on. Cancer, regardless of the species in which it occurs, is the uncontrolled growth of tissue abnormal for the afflicted organism. However, there are substantial differences in cancer transmission dependent on the species. The biggest contrasts in the modes of transmission were revealed between contagious cancer of Syrian hamster, venereal cancer of dogs and analogous malignancy of the Tasmanian devils.

\subsubsection{Contagious cancer in dogs}

\section{Prevalence}

Canine transmissible venereal cancer or venereal sarcoma is the leading cause of death in dogs over the age of two, and risk increases with age. The disease has a worldwide distribution and can be found in many parts of the world. Prevalence varies from relatively high in some geographic regions to rare in others. In some regions it is the most common dog tumor [4].

\section{Transmission}

The disease is usually spread when dogs mate, resulting in the direct physical intrusion of viable cancerous matter from an afflicted dog to a healthy one. The intrusion may also be realized through licking, biting and sniffing tumor-affected areas. Usually the tumors are transmitted dogs sexually. The tumor may also be transplanted from site to site and dog to dog by direct contact with the tumorous mass [15].

\section{Causative agent}

The tumor masses are themselves the infectious agents, the origin of which is unknown. This is considered an infectious disease of dogs caused by a pathogenic lineage of cancerous cells that live as a unicellular pathogen [16]. The malignant tumor cells from one dog are transferred to another $[16,17]$.

\section{Lukewarm immune response against intrusion}

This kind of cancer is an allograft that is transmitted between individuals without immune recognition of the cancerous intruder $[11,15]$.

\section{Clinical exposures}

Individual affliction by the disease arises after natural sexual contact. It is transmitted from animal to animal during copulation and mainly affects the external genitalia (the penis, prepuce, or vulva). Developed tumors can be cauliflower-like, pedunculated, and nodular, papillary, or multilobulated in appearance. They range in size from a small nodule $(5 \mathrm{~mm})$ to a large mass $(>10 \mathrm{~cm})$ that is firm though pliable. The tumor surface is often ulcerated and inflamed and bleeds easily. Tumors may be solitary or multiple and are almost always located on the genitalia. They may be transplanted to adjacent skin and oral, nasal or conjunctival mucosae. The tumor may arise deep within the prepuce or vagina and be difficult to see during cursory examination $[4,18]$.

\section{Progression of disease}

Once on a new victim, the invading cells reproduce over a period of two to six months to form a tumorlike growth, consisting of homogenous populations of large, round cells with distinctive, centrally located nucleoli. Initially, tumors grow rapidly. The canine transmissible tumor undergoes a predictable cycle: the initial growth phase of four to six months, a stable phase, and a non-obligate regression phase. The transmissible venereal tumor of the dog is generally benign. [19]. The spread of malignant cells out of one tumor (metastasis) to other locations is not common 
(5\%) with this type of cancer. Instead of the term "metastasis," the term "variability of the tumor locations" [19] can be used. When it does occur, it is usually to the regional lymph nodes, but kidney, spleen, eye, brain, pituitary, skin and subcutis, mesenteric lymph nodes, and peritoneum may also be targeted [18]. Tumors can regress after one to three months, then the dog can become immune to subsequent re-infection $[3,14]$ probably due to a response from the immunogenic system of the diseased dog [20]. Thus, the immunogenic response of the host can annihilate the tumor's aggressiveness. This is evidence that the tumor is recognized by the immune system as 'non-self.'

\section{Genetics of the canine tumor}

All tumor cells of this type of cancer share an extremely similar genetic code, often, if not always, unrelated to the DNA of their host. The tumors are not genetically related to the infected dog. Canine cancerous cells have fewer chromosomes than normal dog cells. While the karyotype of the normal domestic dog cells has78 chromosomes $(2 n=76$ acrocentric autosomes plus submetacentric $\mathrm{X}$ and $\mathrm{Y}$ sex chromosomes), the chromosome number in canine venereal tumor consist of $2 n=57-59$ chromosomes that that are very different in appearance from normal dog chromosomes [15]. Genetic data strongly indicate that the tumors do not arise from separate cancerous transformation in individual animals [16, 17]. The 37 different tumor samples from seven countries (Mexico, Greece, Israel, Kenya, South Africa, Thailand, and Malaysia) on four continents show very little genetic variation, and the pattern of variation that does exist is consistent with a purely asexual mode of cell transmission. Work on the genetics of the tumors has revealed a number of distinctive features that together suggest that the tumor originated once and subsequently spread worldwide [4]. The genetic and genomic patterns observed are typical of those expected of asexual pathogens, and the extended time since first origin may explain the many remarkable adaptations that have enabled this mammalian cell lineage to live as a unicellular pathogen [4]. Some breeds are more susceptible to certain cancers.

\subsubsection{Transmissible cancer of Tasmanian devils}

\section{Prevalence}

The disease afflicts the Tasmanian devil (Sarcophilus harrisii), a marsupial carnivore endemic to the island of Tasmania. It occurs equally in male and female devils [21]. The disease has spread to almost every corner of the remote island off the southeastern coast of Australia, the only place on Earth where they live in the wild. In the last 10 years, the population has dropped from 150,000 to about 50,000. Nearly 70 percent of the world's Tasmanian devils have been killed in the past 10 years by an infectious cancer called Devil Facial Tumor Disease. The disease has decreased devil numbers by $50 \%$ since its appearance in 1996, with some populations declining by $90 \%$ [22].

\section{Transmission}

Transmission is achieved as devils frequently bite one another on the mouth during mating or while fighting for territory. Most commonly observed in sexually mature individuals of two years or older, the cancer may have actually attached itself to the Tasmanian devils, perhaps via their prey, as a kind of parasite. The disease is transmitted by allograft, whereby an infectious cell line is passed directly between the animals through the bites they inflict on one another $[23,24]$.

\section{Causative agent}

This kind of cancer is a clonally derived allograft, transmitted between devils by the biting they practice when mating or fighting for territory [24]. It appears to be a somatic clonal cell line and may be similar in transmission to canine transmissible venereal tumor and a transmissible sarcoma affecting Syrian hamsters. The prevalence and biology of such somatic cell parasites is generally unknown.

\section{Lukewarm immune response against intrusion}

This tumor is also an allograft that is transmitted between individuals without instant immune recognition of the tumor cells. Any case of transmissible cancer of this kind is induced by physical intrusion of viable tumor cells into a susceptible organism in the form of an allograft not recognized immediately by the immunogenic system as non-self. A colony of Tasmanian devils living in northwestern Tasmania has displayed immunity against the tumor. The nature of this immunity is unknown. It can be either reactive or genetic or both. Researchers supposed that it is provided by the genetic features of the northwestern population that are thought to be genetically distinct from the eastern 
devils [25]. So far, the former have not contracted the cancer again.

\section{Clinical exposure}

Once the cancerous cells infect a Tasmanian devil, the malignant invaders initiate their multiplication, [26] uncontrollable by normal physiological cell regulation. Tissue growth forms a compact malignant oral-facial tumor that fills the animal's mouth and thus destroys the feed. The invasion originates in the Schwann cells, which are vital to the peripheral nervous system. A particular protein, periaxin, which is expressed by these cells, was also present in every tumor [27].

\section{Progression of disease}

The tumor does not evoke an immune response and thus avoids immune rejection during colonization of allogeneic hosts, although experiments have indicated that some Tasmanian devils are normally capable of mounting immune reactions to the allogeneic grafts [25, 26]. The formed tumors obstruct the animal's ability to feed [24]. During disease progression, the tumor ulcerates, becomes friable, and affected devils usually die by starvation within 3-6 months after the first appearance of lesions. The disease kills most but not all Tasmanian devils that catch it. The precise mechanism remains unknown whereby in such cases the progress of intruded allograft is stopped.

\section{Genetics of the tumor}

Alignment of the Tasmanian devil cancer cell line genomes with the genome of healthy animals yielded 691,328 and 699,156 single base substitutions in $87 \mathrm{~T}$ and 53T, respectively, and 317,240 and 307,613 indels in $87 \mathrm{~T}$ and 53T, respectively. The number of variants in the cancer genomes was somewhat higher than the number of variants observed in normal female devil, and in a second normal male genome sequenced to assess normal variation [23]. The chromosomes in these tumors have a complex arrangement identical for every animal studied [24]. The growths were essentially identical to one another but otherwise genetically distinct from the devils themselves. Genetic analysis reveals the parasitic origin of contagious cancer [11]. The malignancy does not have the same DNA as the animals they are killing. The devil cancer genome contains more than 17,000 somatic base substitution mutations and bears the imprint of a distinct mutational process. It is a relatively stable lineage and a high level of genomic instability has not been required for the cancer to become transmissible. The devil cancer genome is relatively stable despite ongoing evolution [23].

Genetic studies indicate that the tumor is clonal and therefore foreign to invaded Tasmanian devils [26].

\subsubsection{Contagious sarcoma of the Syrian hamster}

\section{Prevalence}

The sarcoma is able to afflict various individuals in any observed populations of Syrian hamster [28].

Transmission The spontaneously transplantable tumor can spread from an affected to a healthy hamster by direct physical contact as well as by feed of tumor material [29]. The cancer can also be transmitted from tumor-bearing to healthy hamsters by the mosquito Aedes egypti [12]. The transmission of the tumor by the mosquitoes is considered to be the result of the transfer of viable cells by the mosquito from one animal to the other.

\section{Causative agent}

This tumor is an allograft that is transmitted between individuals without evoking instant immune response to alloantigens. In reality, there are a wide range of individual responses, from no reaction to low or relatively strong responses because in these cases, tumor cells grew in the hosts of various genetic similarities [25].

\section{Clinical exposures}

The spontaneous tumors appear naturally in the vascular and lymphatic systems of Syrian hamsters $[12,13]$. This is a spontaneously transplantable sarcoma with leukemic manifestations. The mass of the tumor consists mainly of reticulum cells of the reticuloendothelial system, [29] which exist constitutionally in lymph nodes, bone marrow and spleen. In lymph nodes, they function as stromatic cells. Endothelial tumors (hemangio-endotheliomas) generally originate in the liver and spleen with similar incidence in both sexes. Among tumors of miscellaneous sites, Harderian gland adenomas predominated in males. Malignant lymphomas develop in higher incidences. Skin neoplasms and tumors of the bone and soft tissue can be present too [28]. 


\section{Progression of disease}

When passed by subcutaneous transplantation, tumor cells appear in the blood after 5 days and just before the death of the hamster reach a concentration greater than 100,000 per mm3 [12]. The transfer of these circulating cells from tumor-bearing hamsters to hamsters without tumors leads to the transmission of the next tumor [13].

\section{Genetics of the tumor}

According to chromosome studies, $[12,13]$ the cells of all tested cancerous animals had identical, very consistent and highly specific tumor karyotype differing from the normal pattern for hamster cells.

\subsubsection{Local conclusion}

Each of most discovered transmissible tumors exploits a relevant animal species (dogs, Tasmanian devils or Syrian hamsters) and thus has its unique adaptations to the host. The transmission of animal cancer is realized mainly by natural sexual relations intrinsic for relevant host species. Furthermore, all of these animal cancers have a common etiology: they are transmitted by the physical transfer of viable cancerous cells. The cells of contagious animal cancers are highly unusual pathogens. They pass between individuals and their inner structures as exceptional infectious agents. The development of individual cancer is initiated in animals by the intrusion into the afflicted body of a deviant cell clone (or clones), inherently immune to normal physiological regulators of cell growth and tissue formation [10]. Animal cancers are also characterized by genetic dissimilarity between cancerous cells and the animals they invade. In other words, any transmissible animal cancer is a result of the intrusion of genetically dissimilar viable somatic material and its subsequent self-reproduction in the invaded living body. The dissimilarity is revealed in the structural and functional traits of the animal's cancer cells. The ability of cancerous cells to perform uncontrollable self-reproduction is the first of these traits and is evidence of the structural immunity of the cells against natural regulation of cell dividing. The subsistence of cancerous cells and tumor masses is provided at the expense of both the structures (proteins, lipids, saccharides) and functions (the supply of oxygen, nutritive substances and means for reproduction) of the invaded organism. Independent of the host species, any transplantable animal cancer is able to suck the lifeblood out of the afflicted body. This is a kind of marauding parasitism performed by the invaded cells of dissimilar animal origin. In any of the animal species discussed, cancer develops without immediate immune recognition of the invading cancerous matter. The cells are allografts for afflicted animal but invade its body with no immune rejection. They are not recognized by the victim immune system as non-self.

\subsection{Non-contagiousness of human cancer}

\subsubsection{New updates to genomic ties of human cancer}

Before the paradigm of the parasitic nature of human cancer was developed, $[7,10]$ there were no known naturally occurring ways for the transmission and spread of cancer in humans. Even the very thought of cancerous invasion between peoples did not correlate with the prevailing hypothesis of a stochastic origin of any cancer out of somatic mutation of a single cell. There were only rare reports of artificial cancer transmission between humans by an accidental transfer of cancer cells through organ transplantation or during surgical procedures as well as the problematic transfer of cancer cells from mother or co-twin via placenta. Only $0.04 \%$ of organ transplant recipients contract cancer from the donor organ. Furthermore, the survival of transplanted cancers in healthy humans was exceedingly rare and documented in only a handful of cases. Genetic immunity probably prevented such cancers from taking hold [10]. But in contrast to contagious animal cancers the cells of human cancer can not pass between individuals as usual infectious agents. Friends, family members and caregivers of cancer patients need not be unduly concerned with the remote possibility of "catching cancer" [3] via direct physical contact with cancer carriers. Human cancer was not generally considered a transmissible disease. Meanwhile, in the middle of the 20th century, cancer overtook many infectious diseases as an important human killer. It became one of the biggest threats to global human health. It takes a terrible and growing 
human toll and its prevalence continues to grow. Meanwhile, like undoubtedly transmissible malignant diseases of animals, human cancer is also characterized by the same set of traits characteristic of malignant growth naturally transmissible among animals. Relevant information of the totality of these traits has recently been summarized and interpreted elsewhere [10]. Undoubted analogies can be seen in the prevalence, clinical exposures, progression of disease, the origin of causative agents, and especially in the genetic deviations characteristic of both animal and human malignancies. Any cancer sustains itself at the expense of substances in the victim's body. This set of traits includes the abnormal reproduction of some aberrant cells and consequent growth of relevant aberrant tissues in different parts of the afflicted organism. Both animal and human cancers are able to exhaust the life- supporting functions of the invaded body and intoxicate its life supporting organs. In contrast to transmissible animal cancers human cancer is unprecedentedly exceptional maneater. Recent studies, [30] together with the set of data discussed above, allow us to suppose that like any other multicellular beings, cancer contains a variety of different cells that are under different genetic regulation and possess different behaviors. Cancer consists of a couple of functionally heterogeneous cell lineages that vary with respect to their distinctive structural or physiological functions and potentials. The heterogeneity within tumor cell lineages may also determine the differences within the kinds of tumors and their locations. Cancer is able to maintain its structural stability through many generations. The diversity of cancer composition remains stable over its sequential long-term propagation [30]. These means that both animal and human cancers have developed many adaptations that enable these aberrant lineages of mammalian cells to exist as a multicellular parasite [7, 8]. At the same time, in contrast to animal cancer, the malignant disease of humans does not possess the ability to transfer its living cells from one person to another.
Human cancer exploits another way to ensure the maintenance of its own life after its host is exhausted and dies.

\subsubsection{Human cancer arises via intrusion a victim genome}

The renewed set of final evidence of the new paradigm allowed confirmation that the descent of human cancer has been predetermined by genome mutations which have created, in evolution, interethnic differences in molecular constitution of intrinsic physiological systems responsible for the regulation of cell dividing and tissue growth. Consequent xenogamous mating between members of such different ethnoses may lead to the intrusion of the descendant's genome with components of deviant genetic information that induce carcinogenesis. The descent and consequent subsistence of human cancer regularly includes obligatory rotation of its successive forms (Table 1). The development of individual cancer is initiated by the appearance of a deviant cell clone inherently immune to autochthonous regulators of cell growth and tissue formation [10]. The cells are able to grow independent of physiological control of autochthonous cell replication. This clone is foreign (non-self) for the afflicted body with many of its traits. The deviant cells appear in a human body as the result of genome transformation performed over the heterozygous crossbreeding between parental gametes with partially different (divergent) genotypes [9]. This is a kind of chimerism or cellular mosaicism, the occurrence in an individual of two or more cell clones of different genome constitutions, derived from different parental individuals [31, 32]. Such heterozygous mosaicism arises as a result of hybridization between two organisms genetically different in some of the relevant traits. For instance, one of them is constitutionally immune to appropriate physiological regulators whereas its mating partner is constitutionally sensitive to it [1]. The heterozygosity results in the coexistence in the offspring's genome of at least two active allelomorphic genes. Both alleles function dominantly and create two allelic cell clones, whose subpopulations are formed and distributed in the body before postnatal ontogenesis. The heterozygous offspring expresses both alleles 
Pure Appl. Bio., 2(1): 7-16, March- 2013.

Table 1. Successive forms of cancer subsistence

\begin{tabular}{|c|c|c|c|c|c|}
\hline Parent & Genomic & \multicolumn{2}{|c|}{ Unicellular forms } & \multicolumn{2}{|l|}{ Multicellular forms } \\
\hline A & $\begin{array}{c}\text { Normal } \\
\text { parent } \\
\text { genome }\end{array}$ & $\begin{array}{l}\text { Normal } \\
\text { gamete }\end{array}$ & \multirow{2}{*}{ Cancerous zygote } & \multirow{2}{*}{$\begin{array}{l}\text { Micro-populations } \\
\text { of cancerous cells }\end{array}$} & \multirow{2}{*}{$\begin{array}{c}\text { Tumors } \\
\text { (developed } \\
\text { populations of cancerous cells) }\end{array}$} \\
\hline B & $\begin{array}{c}\text { Deviant } \\
\text { parent } \\
\text { genome }\end{array}$ & $\begin{array}{l}\text { Deviant } \\
\text { gamete }\end{array}$ & & & \\
\hline
\end{tabular}

equally but in different sizes and in separate locations around the body. Thus, over such xenogamous formation of descendant's zygote, its genome becomes admixed with a block of aberrant, potentially carcinogenic genes. This leads to the intrusion of the offspring's genome with heterozygous genes and the formation in the offspring's body of coexisting cell clones with opposite autochthonous regulators of cell and tissue growth. The emergence of such a cancerous clone and its dispersion around the body in the form of one or more discrete micro-populations is performed before postnatal ontogeny in the manner used to dispose of other embryonic tissues and organs. That is why, despite the genetic and phenetic dissimilarity of human cancer with its host, the lymphatic system of individual adaptive immunity does not recognize the deposited cancer cells as foreign and does not destroy them. After the end of their disposition, the subpopulations continue to reside in their stable places like cell masses of small but different sizes. Cancerous cell populations subsist on life supporting functions provided by the host. Any individual cancer arises and exists as a result of natural ecological relations between two organisms in which the xenogamous one (the consumer) obtains the stuff and energy for its life at the expense of substances composed of the consumed organism (the victim or host). Cancer is a kind of parasitism. The marauding way of life exploited by populations of cancerous cells is performed mainly by their molecular enzymatic agents, targeted either on the splitting of the host's macromolecules or producing functional inhibition of the host cells. At a relevant time of a host's life (mainly after 40 years of age), the uncontrollable growth of such micro-subpopulations becomes visible in the form of detectable extra cell masses of cancerous tissue, the malignant tumors. The largest of the subpopulations achieves the size of detectable tumor far earlier than the smaller ones, thus forming the first appeared cell mass usually called the 'primary' tumor. The growth of all subpopulations of a cancerous clone is controlled by their own united physiological mechanism which maintains the whole structure of cancer within a genetically predetermined size. The destruction of one or more tumors boosts the growth of other subunits of the clone. Various evidence of this intriguing phenomenon has recently been summarized and interpreted elsewhere. [10] Human cancer possesses its own schedule (the program of ontogenesis) as well as the abilities of communication and physiological synchronization between its intra-host subunits. The existence of these intrinsic traits has been initially estimated [7-10] with detailed presentation and discussion of the evidence. Later, it has been experimentally shown that the spread of melanoma in the mouse model correlated with the eventual progress of the disease in human patients. Conversely, melanomas that did not progress after surgical removal of the primary tumors from patients also developed slowly or inefficiently in animals, even after repeated passages of tumor cells through several generations of mice. This finding also demonstrated that the key factors that regulate the rate of cancer and mode of development are intrinsic to the invading cancerous matter [33]. This set of constitutional adaptive traits could be thought to be a result of evolution over many hundreds of millennia. The date of its initiation could be referred, for instance, to regular hybridization and exchange of genes between mutual ancestors of chimps and humans that may have occurred over a few million years [34] as well as to the epoch of xenogamous intercourse of European Homo sapiens with Homo neandertalensis. The last gene flow from Neanderthals (or their relatives) into Europeans likely occurred 37,000-86,000 years ago, and most likely $47,000-65,000$ years ago [35].

\subsubsection{Human cancer transmits via intrusion of genome}

According to the genomic paradigm; both animal and human cancer belong to the group of invasive 
diseases whose subsistence depends on regular transmission of the causative agent from one victim's body to another. Infections and parasitic invasions belong to the group too. Like any other contagious disease, cancer arises and exists as a result of natural ecological relations between two species in which the contagious species (the consumer) obtains the matters and energy for its life, reproduction and subsequent transmission at the expense of substances contained in the victim. These actions exhaust the lifeblood out of afflicted body and thus restrict its vitality, provoking the state of disease and a loss of victim viability. The transmission of cancerous invasive agents inside the body of next victim is mainly carried out by means of the victim's ecological communications, through which the regular physiological function of self-reproduction is provided; (sexual transmission). Human cancer transmits via sexual intercourse. The carrier of human cancer is characterized by a complex of traits necessary for providing the host's ability to transmit deviant genomes into relevant gametes, execute multifold acts of fertilization and breed descendants to the stage that is usually called complete maturity. The absence of any of the abilities sharply diminishes the chances of the cancerous genome to prolong its life in the genomes of descendent generations. The growth of human cancer results in its initial micropopulations becoming visible in the form of tumors mainly after 40 years of age. This may mean human cancer possesses its own schedule of life (the program of ontogenesis) different of those belonging to its victim. Natural selection favors those cancerous cell lineages whose schedule of life did not restrict the reproductive, i.e. transmissive, function of the afflicted person nor its care for its offspring up to the reproductive (transmissive) stage. Saving its own life via self-reproduction is an extraordinarily important function of any form of living matter. Human cancer performs this function very successfully by maintaining long-term propagation. Human cancer, the recently discovered kind of parasitic beings, also developed all relevant adaptations including the ability to circulate between people. This parasitic invader could belong to the Class - Mammalians, Species - Genomeintruder malevolent (G.malevolent). This species developed in evolution not only banal also unique adaptations to its modus vivendi. During the formation of intruded zygote, the deviant components of xenogamous genetic code appear to be included in the formed united genetic code. Since the components continue to exist in it and function over the creation of intruded cells with their plethora of both banal and unique traits. Being implanted in the genome of its current host, the genome of human cancer ensured that it was reproduced in the genomes of children via selfreproduction of the cancer-carrying parent. Like any other components of genetic code, they are able to be reproduced in the descendant genomes and thus multiplied and dispersed between people.

\subsection{Conclusion}

The current pandemic spread of cancer has been brought about by the growing expansion of interethnic admixture favored by growing industrialization, urbanization, globalization, and migration. The currently observed increasing incidence of the disease has been induced by the intensification of xenogamous genetic admixture within ethnically mixed populations. These new notions provide the framework and some initial landmarks for the location of genomic ties and roots of cancer origin and should encourage the appearance of new research ideas and proposals for cancer prevention and therapy. There remains much to be learned about this extraordinarily unique and extremely complex disease. According to the xenogamous paradigm, the search for a coveted clue to the genomic roots of cancer should be oriented on the discovery of structural and functional differences between the genomes of cancerous and normal cells. The initial overall prevention of cancer could be started by the voluntary restriction of xenogamous fertilization as well by the launching of noncancerous genealogies. The risk of cancer development for our children should be at the core of prophylactic doctrine. "Will our children develop cancer?" This tough question should be asked by each couple before they marry. The genealogies of expectant moms and dads must be discovered for the absence of cancer disease among any of their accessible ascendants and genetic relatives. This kind of protective parenting is now on its way to becoming a mainstream medical testing at first by routine genetic methods but finally or in special cases by whole genome sequencing. It is time now to start searching the genomes of every groom and bride in order to assess risk of producing carcinogenic combinations in the genomes of their descendants. Appropriate genomic tests must be performed before conception. The results can provide early warnings about the deadliest and most debilitating disease that may not strike until adulthood. Those warnings can help people either to make rationale decisions about their marital plans or at least to be prepared to enable 
timely treatment and elaborate plans about long-term care.

\section{References}

1. Rumyantsev SN (2008) Hereditary Immunity: Fundamental Principles and Exploitation in Life Study and Health Care. New York, Nova Biomedical Books.

2. Rumyantsev SN (2009) The Discredit of Cancer Metastasis. Science Advisory Board [http://www.scienceboard.net/community/pe rspectives.227.html].

3. Welsh JS (2011) Contagious cancer. Oncologist 16(1):1-4.

4. Rebbeck CA, Thomas R, Breen M, Leroi AM and Burt A (2009) Origins and evolution of a transmissible cancer. Evolution 63(9):2340-2349.

5. Isoda T, Ford AM, Tomizawa D, van Delft FW, De Castro DG, Mitsuiki N, Score J, Taki T, Morio T, Takagi M, Saji H, Greaves M and Mizutani S (2009) Immunologically silent cancer clone transmission from mother to offspring. Proc Natl Acad Sci U S A 106(42):17882-17885.

6. Greaves MF, Maia AT, Wiemels JL and Ford AM (2003) Leukemia in twins: lessons in natural history. Blood 102 (7):2321-2333.

7. Rumyantsev SN (2009) The Uniqueness and Ordinariness of Cancer Origin and Pathogenesis: New Epidemiological, Clinical and Preventive Perspectives. J Clin Med Res 1(1):32-36.

8. Rumyantsev SN (2010) Hypothesis: Towards the origin of cancer epidemics and pathogenesis. Journal of Carcinogenesis 9(2):1-7.

9. Rumyantsev SN (2011) Functions of hereditary immunity and xenogamy in cancer origin and pandemic spread. Open $\mathbf{J}$ of Immunology 1(2):27-40.

10. Rumyantsev SN (2012) Toward the genomic roots of cancer. Journal of Medicine and Medical Sciences 3(10):638-659.

11. Murchison EP (2008) Clonally transmissible cancers in dogs and Tasmanian devils. Oncogene 27(S2):S19-S30.
12. Banfield WG, .Woke PA, Mackay CM and Cooper HL (1965) Mosquito transmission of a reticulum cell sarcoma of hamsters. Science 148:1239-1240.

13. Cooper HL, Mackay CM and Banfield WG (1964) Chromosome studies of a contagious reticulum cell sarcoma of the Syrian hamster. J Natl Cancer Inst 33:691-706.

14. McAloose D and Newton AL (2009) Wildlife cancer: a conservation perspective. Nat Rev Cancer 9(7):517-526.

15. Mello Martins MI, Arial de Souza FF and Gobello C (2005) Canine transmissible venereal tumor: Etiology, pathology, diagnosis and treatment. Recent Advances in Small Animal Reproduction

16. Murgia C, Pritchard JK, Kim SY, Fassati A and Weiss RA (2006) Clonal Origin and Evolution of a Transmissible Cancer. Cell 126(3):477-487.

17. Dingli D and Nowak MA (2006) Cancer biology: infectious tumour cells. Nature 443(7107):35-36.

18. Rogers K, Walker M and Dillon H (1998) Transmissible venereal tumor: a retrospective study of 29 cases. Journal of the American Animal Hospital Association 34(6):463-470.

19. Mannin PJ and Martin PD (1970) Metastasis of Canine Transmissible Venereal Tumor to the Adenohypophysis. Path vet 7:148-152.

20. Stettner $N$, Brenner $O$, Eilam $R$ and Harmelin A (2005) Pegylated liposomal doxorubicin as a chemotherapeutic agent for treatment of canine transmissible venereal tumor in murine models. J Vet Med Sci 67(11):1133-1139.

21. Hawkins CE, Baars C, Hesterman H, Hocking GJ, Jones ME, Lazenby B, Mann D, Mooney N, Pemberton D and Pyecroft S (2006) Emerging disease and population decline of an island endemic, the Tasmanian devil Sarcophilus harrisii. Biol Conserv 131:307-324

22. McCallum H, Jones M, Hawkins $\mathrm{C}$, Hamede $\mathrm{R}$, Lachish S, Sinn DL, Beeton $\mathrm{N}$ and Lazenby B (2009) Transmission dynamics of Tasmanian devil facial tumor disease may 
lead to disease-induced extinction. Ecology 90:3379-3392.

23. Murchison EP, Schulz-Trieglaff OB, Ning Z, Alexandrov LB, Bauer MJ, Fu B, Hims M, Ding Z, Ivakhno S, Stewart C, Ng BL, Wong W, Aken B, White S, Alsop A, Becq J, Bignell GR, Cheetham RK, Cheng W, Connor TR, Cox AJ, Feng ZP, Gu Y, Grocock RJ, Harris SR, Khrebtukova I, Kingsbury Z, Kowarsky M, Kreiss A, Luo S, Marshall J, McBride DJ, Murray L, Pearse AM, Raine K, Rasolonjatovo I, Shaw R, Tedder P, Tregidgo C, Vilella AJ, Wedge DC, Woods GM, Gormley N, Humphray S, Smith G, Hall K, Searle SM, Carter NP and Stratton MR (2012) Genome sequencing and analysis of the Tasmanian devil and its transmissible cancer. Cell 148(4):780-791.

24. Pearse AM and Swift K (2006) Allograft theory: transmission of devil facial-tumour disease. Nature 439(7076):549.

25. Kreiss A, Cheng Y, Kimble F, Wells B, Donovan S, Belov K and Woods GM (2011) Allorecognition in the Tasmanian devil (Sarcophilus harrisii), an endangered marsupial species with limited genetic diversity. PLoS One 6(7):e22402.

26. Siddle HV, Kreiss A, Eldridge MD, Noonan E, Clarke CJ, Pyecroft S, Woods GM and Belov K (2007) Transmission of a fatal clonal tumor by biting occurs due to depleted MHC diversity in a threatened carnivorous marsupial. Proc Natl Acad Sci U S A 104(41):16221-16226.

27. Murchison EP, Tovar C, Hsu A, Bender HS, Kheradpour P, Rebbeck CA, Obendorf D, Conlan C, Bahlo M, Pyecroft S, Kellis M, Marshall Graves JA, Woods GM, Hannon GJ and Papenfuss AT (2010) The Tasmanian devil transcriptome reveals Schwann cell origins of a clonally transmissible cancer. Science 327(5961):8487.

28. Pour P, Mohr U, Althoff J, Cardesa A and Kmoch N (1976) Spontaneous Tumors and Common Diseases in Two Colonies of Syrian Hamsters. IV. Vascular and Lymphatic Systems and Lesions of Other Sites. J Natl Cancer Inst 56(5):949-961.

29. Brindley DC and Banfield WG (1961) A contagious tumor of the hamster. J Natl Cancer Inst 26:949-957.

30. Kreso A, O'Brien CA, van Galen P, Gan O, Notta F, Brown AMK, Ng K, Ma J, Wienholds E, Pollett A, Gallinger S, McPherson J, Mullighan CJ, Shibata D, and Dick JE (2012) Variable Clonal Repopulation Dynamics Influence Chemotherapy Response in Colorectal Cancer. Science 338(6113).

31. Bonnicksen AL (2009) Chimeras, hybrids, and interspecies research: politics and policymaking. Washington, D.C., Georgetown University Press.

32. McLaren A (1976) Mammalian chimaeras. Cambridge, Cambridge Univ Press.

33. Quintana E, Piskounova E, Shackleton M, Weinberg D, Eskiocak U, Fullen DR, Johnson TM and Morrison SJ (2012) Human Melanoma Metastasis in NSG Mice Correlates with Clinical Outcome in Patients. Sci Transl Med 7(4):159.

34. Patterson N, Richter DJ, Gnerre S, Lander ES and Reich D (2006); Genetic evidence for complex speciation of humans and chimpanzees. Nature 441(7097):1103-1108.

35. Sankararaman S, Patterson N, Paabo S, and Reich D (2012) The Date of Interbreeding between Neanderthals and Modern Humans. PLoS Genet 8(10):e1002947. 